( 2021 ФГБУ «НМИЦ ДГОИ

им. Дмитрия Рогачева»

Минздрава России

Поступила 11.05.2021

Принята к печати 12.07.2021

Контактная информация: Серёгина Елена Александровна, научный сотрудник лаборатории клинического гемостаза ФГБУ «НМИЦ ДГОИ

им. Дмитрия Рогачева»

Минздрава России Адрес: 117997, Москва,

ул. Саморы Машела, 1

E-mail: elsereg@inbox.ru
DOI: $10.24287 / 1726-1708-2021-20-3-52-59$

\section{Дисфункция эндотелия у пациентов с наследственным сфероцитозом и $\beta$-талассемией}

\author{
Я.М. Чуйко를. Е.А. Серёгина ${ }^{2,3}$, Т.А. Вуймо ${ }^{2,3}$, А.В. Полетаев², \\ Н.С. Сметанина \\ ${ }^{1}$ ФГАОУ ВО «Российский национальный медицинский университет им. Н.И. Пирогова» \\ Минздрава России, Москва

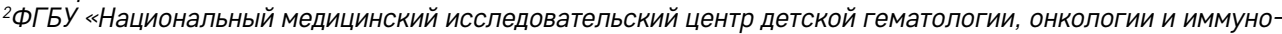 \\ логии им. Дмитрия Рогачева» Минздрава России, Москва \\ ${ }^{3}$ ФГБУН Центр теоретических проблем фризико-химической фрармакологии РАН, Москва
}

Пациенты с наследственным сфероцитозом и $\beta$-талассемией характеризуются повышенным риском тромбоза по сравнению с общей популяцией. Развитие гиперкоагуляции может быть связано с эндотелиальной дисфункцией. Целью данного исследования является оценка состояния свертывания крови и эндотелия у детей с наследственным сфероцитозом и $\beta$-талассемией. Данное исследование одобрено независимым этическим комитетом и утверждено решением ученого совета НМИЦ ДГОИ им. Дмитрия Рогачева. Состояние системы гемостаза у 18 детей с наследственным сфероцитозом ( 10 мальчиков и 8 девочек от 1 до 13 лет) и 8 детей с $\beta$-талассемией (4 мальчика и 4 девочки от 3 до 8 лет) оценивали с использованием стандартных времен свертывания (активированное частичное тромбопластиновое время - АЧТВ, тромбиновое время ТВ, протромбиновый индекс - ПИ), концентрации фибриногена и маркеров дисфункции эндотелия: концентраций эндотелина-1 и тромбомодулина. Пациенты с наследственным сфероцитозом были разделены на 2 подгруппы: во время гемолитического кризиса $(n=11)$ и вне гемолитического кризиса $(n=7)$. Пациентов с $\beta$-талассемией разделили на 3 подгруппы в зависимости от тяжести заболевания: большая, промежуточная и малая формы. Значения АЧТВ, ТВ и ПИ не различались между подгруппами. Мы обнаружили снижение концентрации фибриногена у пациентов с тяжелым течением заболевания: во время гемолитического кризиса при наследственном сфероцитозе (1,9 \pm 0,3 мг/мл при референтном диапазоне 2-3,9 мг/мл) и при большой форме $\beta$-талассемии (1,8 \pm 0,3 мг/мл при референтном диапазоне 2-3,9 мг/мл). Это может быть вызвано потреблением фибриногена при активном гемолизе. Содержание тромбомодулина было повышено у всех пациентов с наследственным сфероцитозом, но медианное значение было выше у пациентов с кризом (6665 пг/мл против 5976 пг/мл при референтном диапазоне 275-909 пг/мл). У пациентов с $\beta$-талассемией содержание тромбомодулина было значительно повышено при большой и промежуточной формах (6389 \pm 537 пг/мл и $6804 \pm 120$ пг/мл соответственно) по сравнению с малой формой $(2727 \pm 213$ пг/мл). Однако и при малой форме $\beta$-талассемии концентрация тромбомодулина все еще была выше нормального диапазона. Концентрация эндотелина-1 была повышена у $55 \%$ пациентов с наследственным сфероцитозом во время кризиса и $43 \%$ больных вне криза. В целом содержание эндотелина-1 было значительнее повышено у пациентов с большой и промежуточной формами $\beta$-талассемии (при малой форме соответствовало референтным значениям) по сравнению с пациентами с наследственным сфероцитозом даже во время криза $(2,33 \pm 2,89$ фммоль/мл и 0,95 $\pm 0,35$ фмоль/мл соответственно). Содержание тромбомодулина и эндотелина-1 выявляет дисфункцию эндотелия у детей с гемолизом, что может быть одной из причин прокоагулянтного состояния. Более резкие изменения наблюдаются при большей интенсивности гемолиза: у пациентов с наследственным сфероцитозом во время гемолитического кризиса и у больных с большой и промежуточной формами $\beta$-талассемии.

Ключевые слова: гемостаз, наследственный сфероцитоз, $\beta$-талассемия, эндотелиальная дисфункция, дети, тромбомодулин, эндотелин-1, гемолиз

Чуйко Я.М. и соавт. Вопросы гематологии/онкологии и иммунопатологии в педиатрии. 2021; 20 (3): 52-59. DOI: $10.24287 / 1726-1708-2021-20-3-52-59$ 


\title{
Endothelial dysfunction in patients with hereditary spherocytosis and $\beta$-thalassemia
}

Ya.M. Chuyko², E.A. Seregina ${ }^{2,3}$, T.A. Vuimo ${ }^{2,3}$, A.V. Poletaev², N.S. Smetanina ${ }^{2}$

${ }^{1}$ Pirogov Russian National Research Medical University of Ministry of Healthcare of the Russian Federation, Moscow

${ }^{2}$ Dmitry Rogachev National Medical Research Center of Pediatric Hematology, Oncology and Immunology of Ministry of Healthcare

of the Russian Federation, Moscow

${ }^{3}$ Center for Theoretical Problems of Physicochemical Pharmacology, Russian Academy of Sciences Moscow

\begin{abstract}
Patients with hereditary spherocytosis and $\beta$-Thalassemia are characterized by the increased risk of thrombosis. The early manifestation of thrombotic complications can occur even in childhood especially after surgery. Hypercoagulability can be associated with endothelial dysfunction. The aim of this study was to investigate the hemostatic state and endothelial function in children with hereditary spherocytosis and $\beta$-thalassemia. The study was approved by the Independent Ethics Committee of the Dmitry Rogachev National Medical Research Center of Pediatric Hematology, Oncology and Immunology. The hemostatic status of 18 children ( 10 boys and 8 girls from 1 to 13 years) with hereditary spherocytosis and of 8 children ( 4 boys and 4 girls from 3 to 8 years) with $\beta$-thalassemia was assessed using clotting times (activated partial thromboplastin time - APTT, thrombin time - TT, prothrombin time PT), fibrinogen levels and markers of endothelium dysfunction: endothelin-1 and thrombomodulin levels. Patients with hereditary spherocytosis were divided into 2 groups: during the hemolytic crisis (11 patients) and without the hemolytic crisis ( 7 patients). Patients with $\beta$-Thalassemia were divided into 3 groups: $\beta$-thalassemia major, $\beta$-thalassemia intermedia and $\beta$-thalassemia minor. APTT, TT and PT were not changed significantly between groups. We find the decreased fibrinogen levels in patients with severe condition: in hereditary spherocytosis patients during hemolytic crisis $(1.9 \pm 0.3 \mathrm{ng} / \mathrm{ml}$ with normal range $2-3.9 \mathrm{ng} / \mathrm{ml})$ and in $\beta$-thalassemia major patients $(1.8 \pm 0.3 \mathrm{ng} / \mathrm{ml}$ with normal range $2-3.9 \mathrm{ng} / \mathrm{ml}$ ). This could be caused by consumption of fibrinogen during acute hemolysis. The Thrombomodulin levels were increased in all hereditary spherocytosis patients, but median value was higher in group with hemolytic crisis $16665 \mathrm{pg} / \mathrm{ml} v \mathrm{vs}$ $5976 \mathrm{pg} / \mathrm{ml}$ with ormal value $275-909 \mathrm{pg} / \mathrm{ml}$ ) indicating endothelium dysfunction and activation of blood clotting. In $\beta$-thalassemia patients Thrombomodulin levels were more elevated in $\beta$-thalassemia major and $\beta$-thalassemia intermedia $(6389 \pm 537 \mathrm{pg} / \mathrm{ml}$ и $6804 \pm 120 \mathrm{pg} / \mathrm{ml})$ compared to $\beta$-thalassemia minor $(2727 \pm 213 \mathrm{pg} / \mathrm{ml})$ which is still higher than normal range. Endothelin-1 levels were elevated on $55 \%$ with hereditary spherocytosis patients during crisis vs $43 \%$ without. In general Endothelin-1 levels were more elevated in $\beta$-thalassemia patients (were normal in $\beta$-thalassemia minor) vs hereditary spherocytosis patients $(2.33 \pm 2.89 \mathrm{fmol} / \mathrm{ml}$ vs $0.95 \pm 0.35 \mathrm{fmol} / \mathrm{ml})$. Thrombomodulin and endothelin-1 levels revealed endothelium dysfunction in children with hemolysis. More dramatic changes observed in severe condition: in hereditary spherocytosis patients during hemolytic crisis and in $\beta$-thalassemia major and $\beta$-thalassemia intermedia patients.

Key words: hemostasis, hereditary spherocytosis, $\beta$-thalassemia, endothelial dysfunction, children, thrombomodulin, endothelin-1, hemolysis
\end{abstract}

Chuyko Ya.M., et al. Pediatric Hematology/Oncology and Immunopathology. 2021; 20 (3): 52-59.

DOl: $10.24287 / 1726-1708-2021-20-3-52-59$

аследственный сорероцитоз (НС) и $\beta$-талассемия - это наследственные гемолитические анемии, которые объединяет общий синдром внутриклеточный гемолиз. Однако механизм возникновения гемолиза различный. В основе патогенеза HC лежит аномалия каркасных белков мембраны эритроцита, в связи с чем нарушается эластичность этих клеток. При $\beta$-талассемии вследствие дисбаланса глобиновых цепей происходит окислительное повреждение внутреннего слоя клеточной мембраны с нарушением деформируемости эритроцитов. Эти изменения, как при НС, так и при $\beta$-талассемии, приводят к элиминации эритроцитов из кровотока.

Клиническая картина у пациентов с гемолитической анемией разнообразна: от бессимптомного носительства, хронического компенсированного гемолиза и до тяжелой гемолитической анемии, требующей заместительных трансфузий донорских эритроцитов, а иногда и спленэктомии. Многие исследователи обращают внимание на нарушения в системе свертывания крови, которые могут приводить к различным осложнениям, в том числе к тромбозам, кардиологическим нарушениям и легочной недостаточности [1-8].

Механизмы, сопутствующие гиперкоагуляции при большой и промежуточной формах $\beta$-талассемии, включают в себя активацию тромбоцитов,
(C) 2021 by «D. Rogachev NMRCPHO|» Received 11.05.2021 Accepted 12.07.2021

\author{
Correspondence: \\ Elena A. Seregina, \\ a Research Associate of the Laboratory \\ of Clinical Hemostasis, Dmitry Rogachev \\ National Medical Research Center \\ of Pediatric Hematology, Oncology and \\ Immunology, Ministry of Healthcare \\ of the Russian Federation \\ Address: 1 Samory Mashela St. \\ Moscow 117997, Russia \\ E-mail: elsereg@inbox.ru
}

аномальную экспрессию молекул адгезии на эндотелиальных клетках сосудов, дисфункцию печени, повышение количества прокоагулянтных поверхностей и циркулирующих микровезикул в связи с аномалией эритроцитов [6, 9-13]. Гиперкоагуляция у пациентов с этими формами $\beta$-талассемии может наблюдаться с детского возраста [14].

Несмотря на различные механизмы возникновения гемолиза, при НС гиперкоагуляцию связывают с аналогичными, что и при $\beta$-талассемии, причинами, это активация тромбоцитов, наличие микровезикул, высвобождающихся в процессе лизиса эритроцитов, эндотелиальные нарушения и расстройство микроциркуляции [1, 2, 4, 5, 15-18]. Тем не менее основной механизм развития гиперкоагуляции при $\beta$-талассемии и НС остается в значительной степени неясным.

Риск развития тромбоза при гемолитических анемиях возрастает после хирургических вмешательств, в особенности - спленэктомии $[18,19]$.

До сих пор одним из вопросов остается возможность оценки функции эндотелия с точки зрения нарушений системы гемостаза. Эндотелиальные клетки выстилают кровеносные сосуды и играют важную роль в том числе и в гемостазе. Эндотелий содержит в себе ряд трансмембранных белков и рецепторов, а также способен накапливать и синте- 
зировать большое количество биологически активных веществ, участвующих в системе свертывания крови. Последние исследования показывают, что нарушение нормальной функции эндотелия напрямую приводит к развитию сердечно-сосудистых заболеваний [20].

Эндотелиальная дисфункция может играть не последнюю роль в нарушении системы гемостаза у детей с гемолитическими анемиями. Традиционно используемые коагулологические методы диагностики состояния свертывающей системы, за исключением концентрации фибриногена [5], не обладают достаточной чувствительностью для характеристики механизмов изменения свертывания крови при гемолитических анемиях, однако сообщается о гиперкоагуляции, детектируемой с помощью интегральных тестов гемостаза $[21,22]$. Тем не менее вопрос о механизме развития этих нарушений и вкладе в него эндотелиальной функции остается открытым. Данная работа посвящена оценке состояния эндотелия у детей с НС и $\beta$-талассемией.

\section{МАТЕРИАЛЫ И МЕТОДЫ ИССЛЕДОВАНИЯ}

Настоящее исследование одобрено независимым этическим комитетом (выписка из протокола №10/2014) и утверждено решением ученого совета ФГБУ «НМИЦ ДГОИ им. Дмитрия Рогачева» Минздрава России.

\section{Пациенты}

В исследование включены 18 пациентов с НС (10 мальчиков и 8 девочек в возрасте от 1 до 13 лет, медиана возраста 6 лет), все до проведения спленэк-

\section{таблица}

Концентрация гемоглобина, билирубина и активность лактатдегидрогеназы у пациентов с НС и $\beta$-талассемией Table

The levels of hemoglobin and bilirubin and the activity of lactate dehydrogenase in patients with hereditary spherocytosis (HS) and $\beta$-thalassemia

\begin{tabular}{|c|c|c|c|c|}
\hline $\begin{array}{l}\text { Параметр } \\
\text { Parameter }\end{array}$ & $\begin{array}{c}\text { Гемоглобин, г/л } \\
\text { (норма 115-138 г/л) } \\
\text { Hemoglobin, g/L (normal } \\
\text { range: } \\
115-138 \mathrm{~g} / \mathrm{L} \text { ) }\end{array}$ & $\begin{array}{l}\text { Билирубин общий, } \\
\text { мкмоль/л (норма } \\
1-17 \text { мкмоль/л) } \\
\text { Total bilirubin, нmol/L } \\
\text { (normal range: } \\
1-17 \mu \mathrm{mol} / \mathrm{L} \text { ) } \\
\end{array}$ & $\begin{array}{c}\text { Билирубин прямой, } \\
\text { мкмоль/л (норма } \\
0-3,4 \text { мкмоль/л) } \\
\text { Direct bilirubin, } \mu \mathrm{mol} / \mathrm{L} \\
\text { (normal range: } 0-3.4 \text { mol/L) }\end{array}$ & $\begin{array}{c}\text { Лактатдегидрогеназа, } \\
\text { Ед/л (норма } \\
100-280 \text { Eд/л) } \\
\text { Lactate dehydrogenase, U/L } \\
\text { (normal range: } \\
100-280 \text { U/L) }\end{array}$ \\
\hline \multicolumn{5}{|c|}{$\begin{array}{l}\mathrm{HC} \\
\mathrm{HS}\end{array}$} \\
\hline $\begin{array}{l}\text { Состояние криза } \\
\text { During a crisis }\end{array}$ & 58-116 & $16-279$ & $4,2-10,8$ & $288-3156$ \\
\hline $\begin{array}{l}\text { Вне криза } \\
\text { Without a crisis }\end{array}$ & $104-166$ & $6-83$ & $2,4-8,2$ & $155-271$ \\
\hline $\begin{array}{l}\text { Статистическая } \\
\text { значимость различий } \\
\text { Statistical significance of } \\
\text { differences }\end{array}$ & $p=0,02$ & $p=0,04$ & $p=0,03$ & $p=0,001$ \\
\hline \multicolumn{5}{|c|}{$\begin{array}{c}\beta \text {-талассемия* } \\
\beta \text {-thalassemia* }\end{array}$} \\
\hline $\begin{array}{l}\text { Большая форма } \\
\beta \text {-thalassemia major }\end{array}$ & $64-99$ & $28-51$ & $9-13$ & $194-319$ \\
\hline $\begin{array}{l}\text { Промежуточная форма } \\
\beta \text {-thalassemia intermedia }\end{array}$ & $93-113$ & $20-37$ & $6-13$ & $265-317$ \\
\hline $\begin{array}{l}\text { Малая форма } \\
\beta \text {-thalassemia minor }\end{array}$ & $107-119$ & $10-22$ & $4-6$ & $224-236$ \\
\hline
\end{tabular}

томии. Пациенты были разделены на 2 подгруппы в зависимости от интенсивности гемолиза: в состоянии гемолитического криза (11 пациентов, из них 6 мальчиков и 5 девочек в возрасте от 1 до 11 лет) и вне гемолитического криза (7 пациентов, из них 4 мальчика и 3 девочки в возрасте от 2 до 13 лет). Диагноз НС у всех пациентов был установлен в соответствии с клиническими рекомендациями [23]. В исследование также были включены пациенты с $\beta$-талассемией: 4 - с большой формой (2 мальчика и 2 девочки от 3 до 7 лет, медиана возраста 4 года), 2 - с промежуточной формой (1 мальчик 6 лет и 1 девочка 7 лет), 2 - с малой формой (1 мальчик 6 лет и 1 девочка 8 лет). Клинико-лабораторная характеристика пациентов представлена в таблице. Пациенты с большой формой $\beta$-талассемии не получали адекватную заместительную терапию донорскими эритроцитами (гемоглобин до трансфузии у всех пациентов был менее 85 г/л), что сопровождалось сохранением интенсивного гемолиза, выраженной гепатоспленомегалией. На исследование кровь у этих детей забиралась перед началом очередной заместительной трансфузии (примерно через 4-6 нед после проведенной трансфузии). Пациентам с промежуточной формой $\beta$-талассемии проводили фармакологическую стимуляцию синтеза фетального гемоглобина, что обеспечивало субнормальное содержание гемоглобина в кровотоке.

Для определения референтных значений проводилось исследование концентрации тромбомодулина и эндотелина-1 у 20 здоровых взрослых добровольцев (5 мужчин и 15 женщин в возрасте от 18 до 30 лет, медиана возраста 24 года). 


\section{Реагенты}

В исследовании были использованы следующие реагенты: SynthASil, RecombiPlasTin 2G, QFA-Fibrinogen, Thrombin Time (HemosIL, Instrumentation Laboratory, Massachusetts, CШA), набор реагентов Endothelin-1 Elisa kit, (BIOMEDICA, Австрия), набор реагентов Trombomodulin Elisa kit (Cloud Clone, США).

\section{Забор крови и пробоподготовка}

Кровь у пациентов забиралась в пробирку Monovette 3 мл с 0,106М буфера цитрата натрия (pH 5,5) в соотношении объема крови к антикоагулянту 9:1. Кровь была обработана путем центрифугирования при $1600 \mathrm{~g} \mathrm{в} \mathrm{течение} 15$ мин для получения бедной тромбоцитами плазмы (БТП). Часть БТП была заморожена для выполнения исследований.

\section{Клоттинговые тесты}

Следующие тесты были проведены на свежей БТП: активированное частичное тромбопластиновое время (АЧТВ), протромбиновый индекс (ПИ), тромбиновое время (ТВ), концентрация фибриногена. Все исследования проводились в соответствии с инструкциями соответствующего производителя на полуавтоматическом анализаторе ACL TOP 700 (Instrumentation Laboratory, США) в лаборатории клинического гемостаза НМИЦ ДГОИ им. Дмитрия Рогачева. Диапазон нормальных значений предоставлен производителем.

\section{Концентрация эндотелина-1 и тромбомодулина}

Тесты были проведены на размороженной БТП методом иммуноферментного анализа. Для измерений использован планшетный ридер ThermoMax UV Max Kinetic Microplate reader (США).

\section{Статистический анализ}

Анализ статистических различий между рядами данных проводился с использованием критерия Манна-Уитни с уровнем статистической значимости $p<0,05$. Для всех расчетов была использована программа OriginPro 8,0 (OriginLab Corporation, США).

\section{РЕЗУЛЬТАТЫ ИССЛЕДОВАНИЯ}

Стандартный тест оценки внешнего пути свертывания крови, ПИ по Квику (в процентах) оставались в диапазоне нормальных значений у всех пациентов с НС и $\beta$-талассемией, независимо от тяжести течения заболевания (рисунки $1 A, 2 A$ ). В то время как АЧТВ, оценивающее внутренний путь свертывания крови, было удлинено у 2 детей с НС вне криза (рисунок 1Б). Также нами отмечено удлинение ТВ и снижение концентрации фрибриногена у большин- ства пациентов с гемолизом (рисунки 1B, Г, 2B, Г). Несмотря на то, что статистически значимой разницы ( $p>0.05)$ в концентрации фибриногена между группами больных не наблюдалось, что может быть связано с небольшой выборкой, у 67\% пациентов с НС во время криза и у 75\% больных с большой формой $\beta$-талассемии отмечено снижение фибриногена, в то время как вне криза НС только в 14\% случаев наблюдалось аналогичное снижение фибриногена.

Некоторые исследователи также описывают снижение концентрации фибриногена у пациентов с гемолитической анемией $[5,22,24]$, объясняя этот феномен потреблением белка в процессах патологического свертывания при гемолизе [24, 25]. Полученные нами данные о нормальном содержании фибриногена у пациентов вне острого или хронического гемолиза и его снижение при тяжелом течении заболевания подтверждают высказанную ранее гипотезу. ТВ часто напрямую зависит от концентрации фибриногена и в связи с самой низкой активацией среди времен свертывания более чувствительно даже к минорному потреблению факторов свертывания, которое может возникать на фоне гемолиза и гиперкоагуляции у пациентов. Показатели стандартной коагулограммы не отражают изменений в системе свертывания у пациентов с гемолизом в виде гиперкоагуляции, однако, возможно, отражают итог: некую легкую коагулопатию потребления факторов во время гемолиза в виде легкого удлинения ТВ и снижения фибриногена.

Результаты измерения маркеров эндотелиальной дисфункции, тромбомодулина и эндотелина-1 представлены на рисунке 3. Диапазон референтных значений получен по результатам исследования концентрации эндотелина-1 (0-1 фмоль/мл) и тромбомодулина (315-909 пг/мл) у 20 здоровых добровольцев от 18 до 30 лет. У пациентов с НС наблюдается повышение тромбомодулина как на фоне криза, так и вне его, однако медианное значение во время криза выше, чем вне криза (6665 пг/мл против 5976 пг/мл, $p=0,20$ ), возможно, с увеличением числа исследуемых больных значимость возрастет. Интересно, что у пациентов с хроническим гемолизом, с большой и промежуточной формами $\beta$-талассемии нами отмечено значение тромбомодулина в 3 раза выше, чем у больных с малой формой $\beta$-талассемии (рисунок 3Б). К сожалению, оценить статистическую значимость сложно вследствие малых выборок. Концентрация эндотелина-1 повышена у всех пациентов с НС, однако во время криза концентрация эндотелина-1 почти в 2 раза выше, чем вне криза (медиана 0,9 фмоль/мл и 0,5 фмоль/мл соответственно). У пациентов с большой и промежуточной формами $\beta$-талассемии наблюдались подъемы концентрации эндотелина-1 до 8 фМ/мл, в то время как при малой форме $\beta$-талассемии она 
Рисунок 1

Показатели стандартных тестов оценки свертывания крови у пациентов с НС: А - ПИ по Квику; Б - АЧТВ; В TB; Г - концентрация фрибриногена

НС криз - пациенты с НС в состоянии криза; НС вне криза - пациенты с НС вне криза. Диапазон нормальных значений отмечен штриховкой

Figure 1

Standard coagulation tests in patients with hereditary spherocytosis: A -prothrombin time (PT); Б - activated partial thromboplastin time (aPTT); B - thrombin time (TT); $\Gamma$ - fibrinogen concentration

HS with a crisis: patients with HS experiencing a crisis; HS without a crisis: patients with HS without a crisis. The normal range is shown as hatched
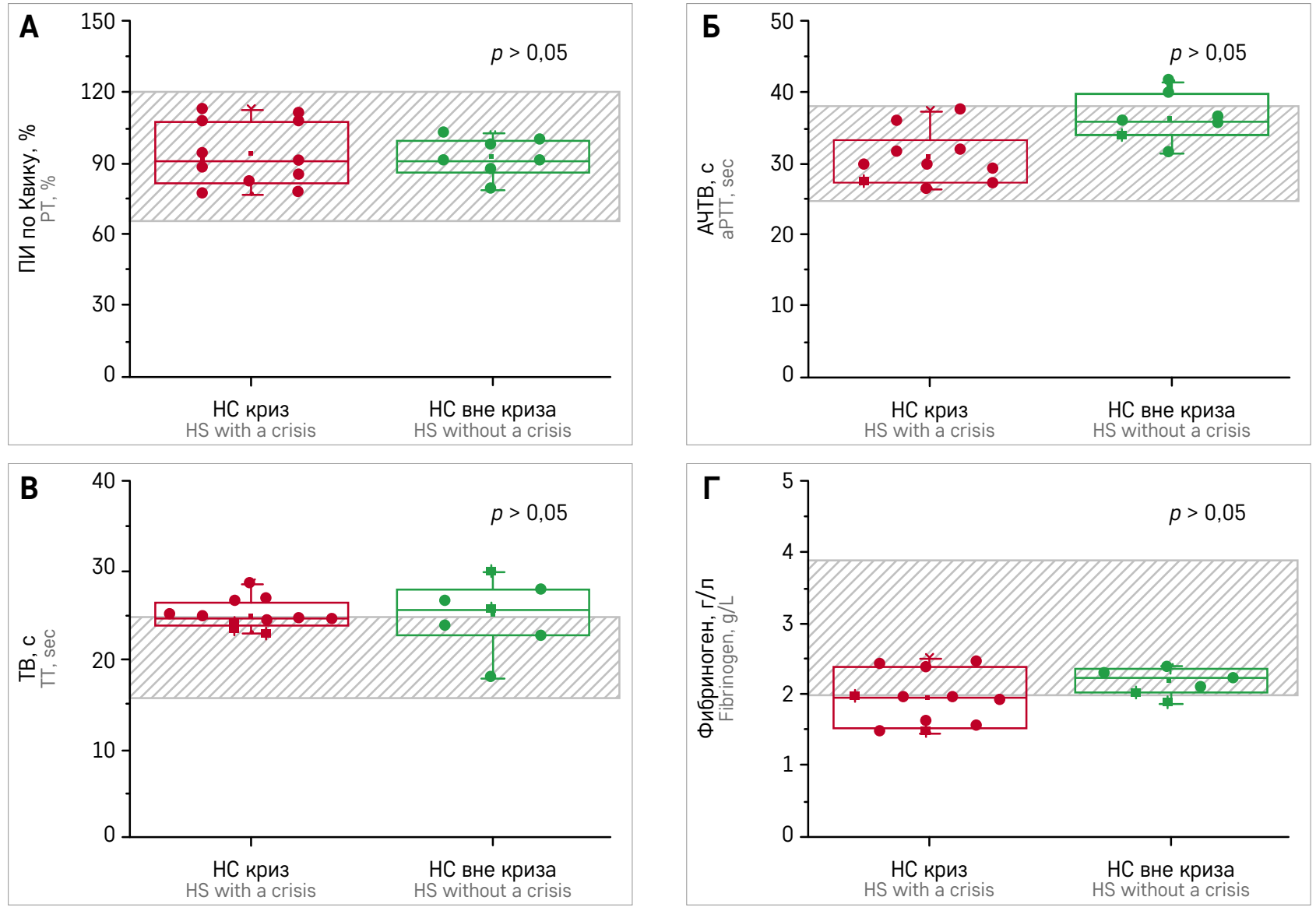

сохранялась в референтных пределах. Полученные нами результаты демонстрируют, что, несмотря на эффективность проводимой фармакологической стимуляции синтеза фетального гемоглобина у пациентов с промежуточной формой $\beta$-талассемии в виде сохранения концентрации гемоглобина более 90 г/л и минимальной спленомегалии, сохраняющийся гемолиз поддерживает напряженность системы гемостаза и заинтересованность эндотелия.

Считается, что в норме есть прямая зависимость между сосудосуживающими и сосудорасширяющими агентами, в том числе между концентрацией NO и поступлением эндотелина-1 в кровоток [26]. Возможно, вследствие снижения содержания NO у пациентов с гемолитическими анемиями [27] мы видим такие изменения в концентрации эндотелина-1, что еще раз указывает на развитие эндотелиальной дисфункции при гемолизе.

Тем не менее тромбозы у детей с гемолитическими анемиями встречаются нечасто и в основном

после оперативного вмешательства [18, 28, 29], однако уже в детском возрасте мы можем наблюдать развитие дисфункции эндотелия - изменения в концентрациях тромбомодулина и эндотелина-1.

\section{ОБСУЖДЕНИЕ РЕЗУЛЬТАТОВ ИССЛЕДОВАНИЯ}

У части пациентов с НС и $\beta$-талассемией могут возникать тромбозы (чаще всего после спленэктомии в случае НС или при промежуточной форме $\beta$-талассемии), особенно в старшем возрасте [14, $18,19]$. Несмотря на то, что первопричина развития гемолитических процессов у этих двух заболеваний разная, основные причины развития тромбозов могут быть схожи (например, активация плазменного и тромбоцитарного звена, эндотелиальная дисфункция и нарушение микроциркуляции) [1, 2, 4, 5, 15-18]. Измененные эритроциты, потерявшие свою пластичность из-за изменения структуры мембраны, при прохождении через капилляры разрушаются, 
Рисунок 2

Показатели стандартных тестов оценки свертывания крови у пациентов с $\beta$-талассемией: А - ПИ по Квику; Б АЧТВ; В - ТВ; Г - концентрация фиибиногена

БФ $\beta$ - большая форма $\beta$-талассемии; ПФ $\beta Т$ - промежуточная форма $\beta$-талассемии; МФ $\beta T$ - малая фрорма $\beta$-талассе-

мии. Диапазон нормальных значений отмечен штриховкой

Figure 2

Standard coagulation tests in patients with $\beta$-thalassemia: A - PT; $5-$ aPTT; B $-\mathrm{TT} ;\ulcorner-$ fibrinogen concentration.

$\beta$-TM - $\beta$-thalassemia major; $\beta$-TI - $\beta$-thalassemia intermedia; $\beta$-TMI - $\beta$-thalassemia minor. The normal range is shown as hatched
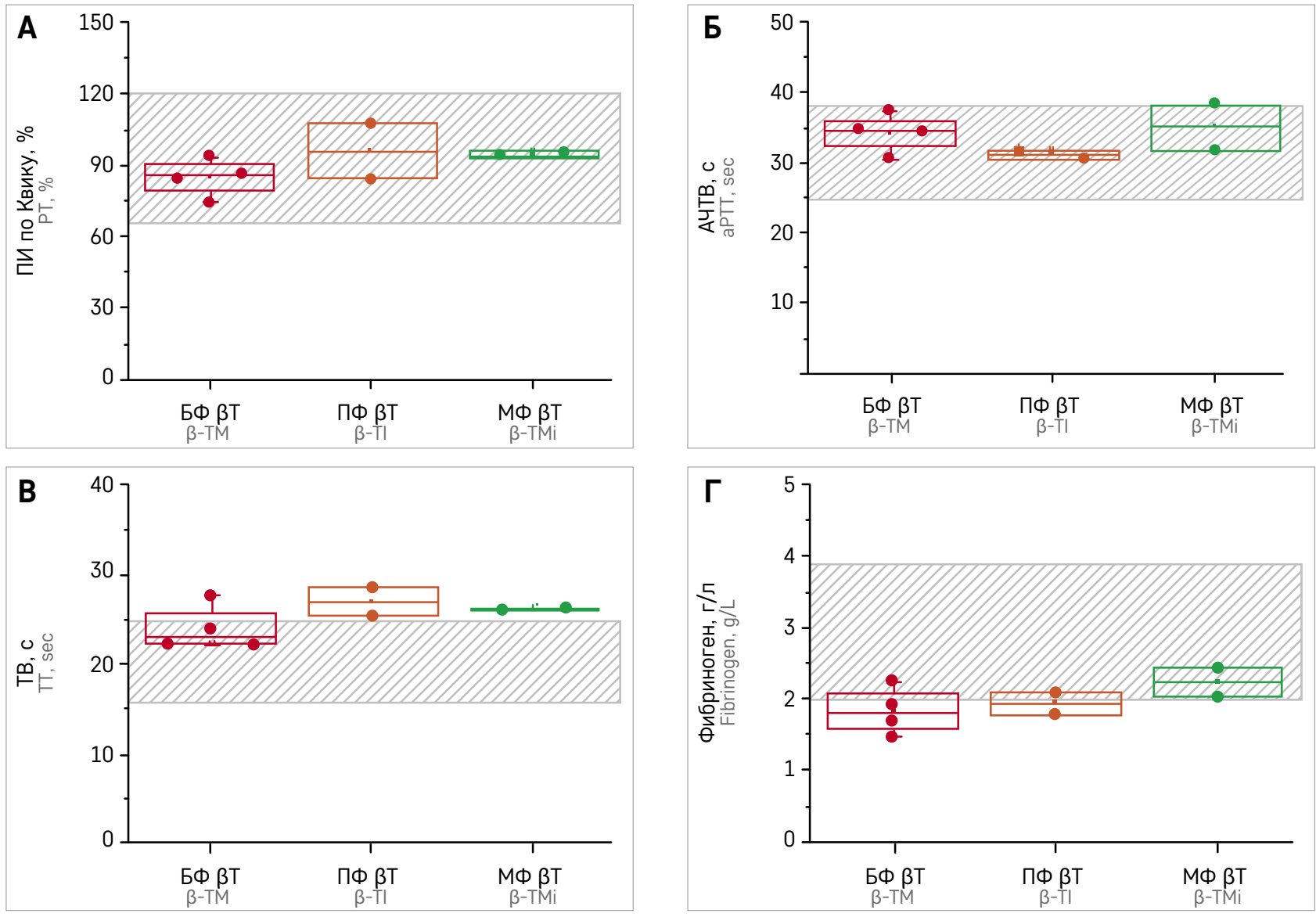

усиливается утилизация эритроцитов макроффагами селезенки, что в совокупности приводит не только к возникновению гемолиза, но и к нарушению свертывания крови. Полученные нами данные показывают вклад в развитие тромбозов такого явления, как дисфункция эндотелия. Эндотелий выстилает поверхность сосудов и при отсутствии повреждений обладает атромбогенными свойствами. При НС и $\beta$-талассемии наблюдается аномальный рост концентраций эндотелина-1 и свободного тромбомодулина, одного из важнейших регуляторов свертывания крови, находящегося на поверхности эндотелия и тысячекратно ускоряющего активацию протеина С при взаимодействии с ним тромбина. Эндотелин же в свою очередь также является важным звеном в поддержании гемостаза, будучи одним из сильнейших агентов вазоконстрикции. Исходя из полученных результатов, можно предположить, что у детей с $\beta$-талассемией и НС развивается дисфункция эндотелия, из-за чего также нарушается гемостатический баланс, что в свою очередь может приводить

к развитию тромбозов при возникновении дополнительных факторов риска (в детской популяции это в основном оперативные вмешательства). До конца механизм развития эндотелиальной дисфункции до сих пор не ясен [30]. Однако в последнее время все больше исследований посвящено маркерам развития эндотелиальных нарушений. Измерение уровней асимметричного диметиларгина [31] у пациентов с большой формой $\beta$-талассемии предсказывает дальнейшее развитие дисфункции эндотелия, снижение содержания NO и простогландина E2 [30] в свою очередь также отражает нарушения в функции сосудистой стенки у пациентов с гемолизом. Сообщается, что в конечном счете эндотелиальные нарушения у таких пациентов приводят к сердечно-сосудистым заболеваниям, что также увеличивает риск развития клинических проявлений нарушений гемостаза [20]. Стандартные методы оценки гемостаза (АЧТВ, ТВ, ПИ по Квику) при этом оказались нечувствительными к протромботическим тенденциям при данных заболеваниях. Однако при усилении гемолиза наблюда- 
Рисунок 3

Концентрация тромбомодулина и эндотелина-1 у пациентов с НС и с $\beta$-талассемией

Диапазон нормальных значений отмечен штриховкой

Figure 3

The concentrations of thrombomodulin and endothelin- 1 in patients with HS and in patients with $\beta$-thalassemia

The normal range is shown as hatched
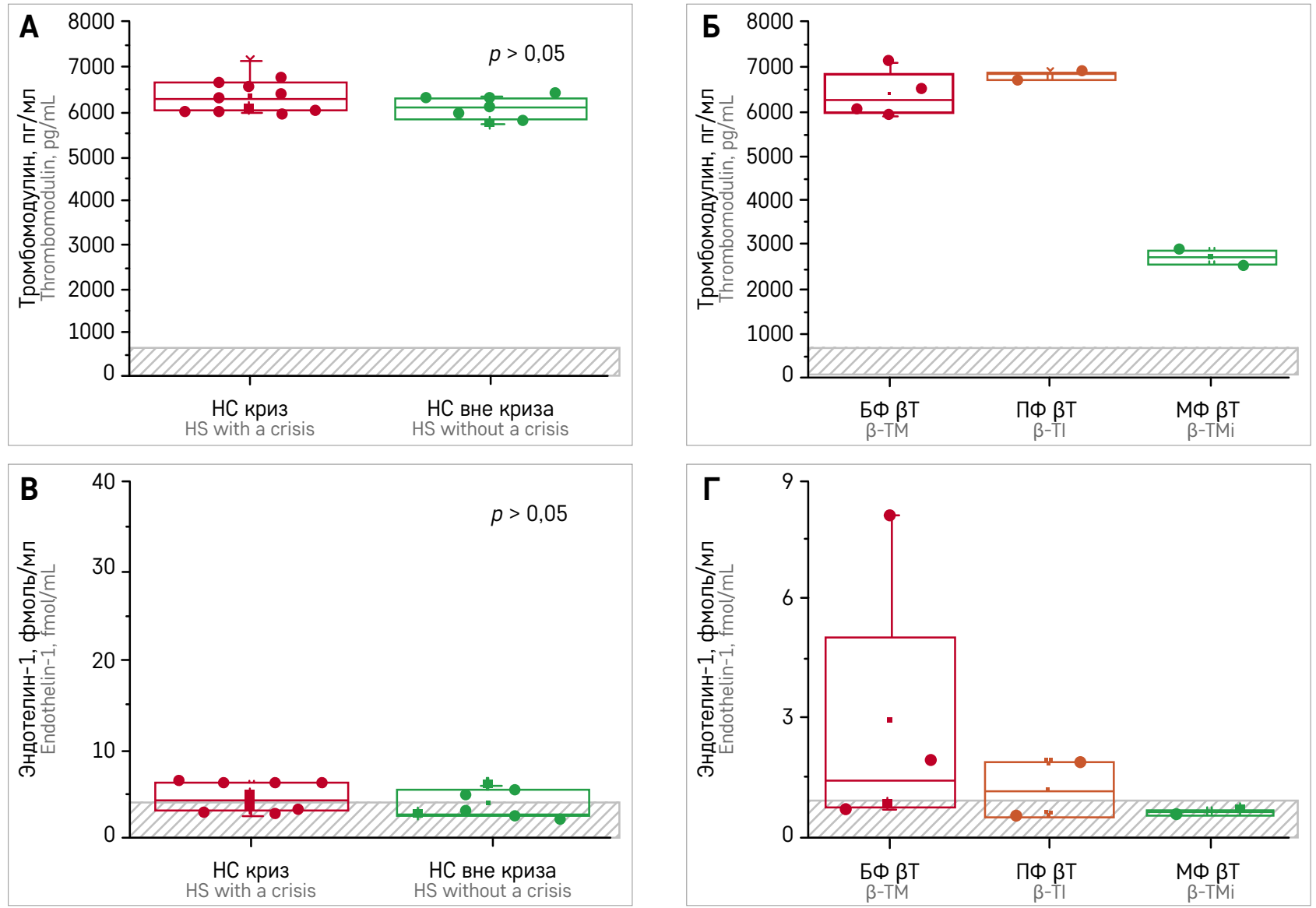

ется снижение концентрации фиибриногена [5, 22, 24]. Изменения маркеров дисфункции эндотелия раньше освещают один из механизмов, приводящих в дальнейшем к нарушению свертывания крови, и, возможно, совместно с другими методиками позволят выявить у пациента риск развития тромботических осложнений.

\section{ЗАКЛЮЧЕНИЕ}

Выявленные нами тенденции в изменении маркеров эндотелиальной дисфункции говорят о развитии нарушений в системе гемостаза у пациентов с гемолизом еще в детском возрасте. Вопрос о механизмах развития эндотелиальной дисфункции при этих патологиях остается открытым, однако полученные данные по изменению тромбомодулина и эндотелина-1 в совокупности с известной клинической картиной гиперкоагуляции не оставляют

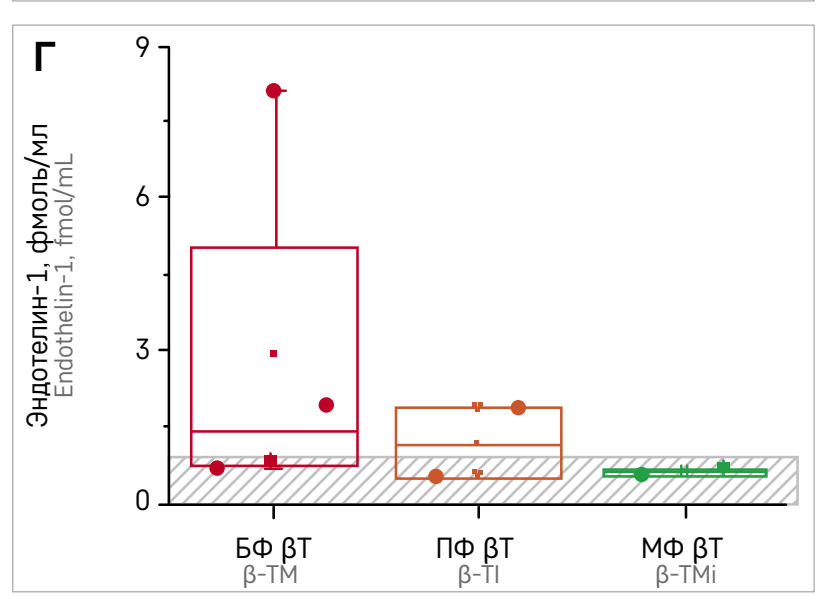

сомнений в участии сосудистого звена свертывания в этих изменениях. Поиск новых маркеров детекции нарушений свертывания и изучение механизмов развития этих нарушений у пациентов с гемолитическими анемиями позволят в дальнейшем рано выявлять повышение риска тромбозов у пациентов с НС и $\beta$-талассемией.

\section{ИСТОЧНИК ФИНАНСИРОВАНИЯ}

Не указан.

\section{КОНФЛИКТ ИНТЕРЕСОВ}

Авторы статьи подтвердили отсутствие конфликта интересов, о котором необходимо сообщить.

ORCID

Chuyko Ya.M. ORCID: https://orcid.org/0000-0003-3650-0309 Seregina E.A. ORCID: https://orcid.org/0000-0002-7534-3863

Vuimo T.A. ORCID: https://orcid.org/0000-0003-3491-1884

Poletaev A.V. ORCID: https://orcid.org/0000-0001-5209-2099

Smetanina N.S. ORCID: https://orcid.org/0000-0002-8805-1499 


\section{Литература}

1. Barker J.E., Wandersee N.J. Thrombosis in Heritable Hemolytic Disorders: Curr Opin Hematol 1999; 6 (2): 71. DOI: 10.1097/00062752-199903000-00003

2. McGrew W., Avant G.R. Hereditary Spherocytosis and Portal Vein Thrombosis. J Clin Gastroenterol 1984; 6 (4): 381-2.

3. Ezov N., Levin-Harrus T., Mittelman M., Redlich M., Shabat S., Ward S.M., et al. A Chemically Induced Rat Model of Hemolysis with Disseminated Thrombosis. Cardiovasc Toxicol 2002; 2 (3); 181-93. DOI: 10.1007/s12012-0020003-6

4. Ataga K.I. Hypercoagulability and Thrombotic Complications in Hemolytic Anemias. Haematologica 2009; 94 (11): 1481-4. DOI: 10.3324/haematol.2009.013672

5. Crary S.E., Troendle S., Ahmad N., Buchanan G.R. Traditional Laboratory Measures of Cardiovascular Risk in Hereditary Spherocytosis. Pediatr Blood Cancer 2010; 55 (4): 684-9. DOI: 10.1002/pbc. 22640

6. Taher A., Isma'eel H., Mehio G., Bignamini D., Kattamis A., Rachmilewitz E.A., et al. Prevalence of Thromboembolic Events among 8860 Patients with Thalassaemia Major and Intermedia in the Mediterranean Area and Iran. Thromb Haemost 2006; 96 (4): 488-91.

7. Bolton-Maggs P.H.B., Stevens R.F., Dodd N.J., Lamont G., Tittensor P. King M.-J. General Haematology Task Force of the British Committee for Standards in Haematology. Guidelines for the Diagnosis and Management of Hereditary Spherocytosis. $\mathrm{Br} \mathrm{J}$ Haematol 2004; 126 (4): 455-74. DOI: 10.1111/j.1365-2141.2004.05052.x

8. Abdullah F., Zhang Y., Camp M., Rossberg M.I., Bathurst M.A., Colombani P.M., et al. Splenectomy in Hereditary Spherocytosis: Review of 1657 Patients and Application of the Pediatric Quality Indicators. Pediatr Blood Cancer 2009; 52 (7): 834-7. DOI: 10.1002/pbc. 21954

9. Ataga K.I., Cappellini M.D., Rachmilewitz E.A. $\beta$-Thalassaemia and Sickle Cell Anaemia as Paradigms of Hypercoagulability. $\mathrm{Br} \mathrm{J}$ Haematol 2007; 139 (1): 3-13. DOI: 10.1111/j.13652141.2007.06740.x

10. Cappellini M.D., Poggiali E., Taher A.T., Musallam K.M. Hypercoagulability in $\beta$-Thalassemia: A Status Quo. Expert Rev Hematol 2012; 5 (5): 505-11, quiz 512. DOI: $10.1586 /$ ehm. 12.42

11. Borenstain-Ben Yashar V., Barenholz Y., Hy-Am E., Rachmilewitz E.A., Eldor A. Phosphatidylserine in the Outer Leaflet of Red Blood Cells from Beta-Thalassemia Patients May Explain the Chronic Hypercoagulable State and Thrombotic Episodes. Am J Hematol 1993; 44 (1): 63-5. DOI: 10.1002/ajh.2830440114
12. Panigrahi I., Agarwal S. Thromboembolic Complications in Beta-Thalassemia: Beyond the Horizon. Thromb Res 2007; 120 (6): 783-9. DOI: 10.1016/j.thromres.2007.01.015

13. Habib A., Kunzelmann C., Shamseddeen W., Zobairi F., Freyssinet J.-M. Taher A. Elevated Levels of Circulating Procoagulant Microparticles in Patients with Beta-Thalassemia Intermedia. Haematologica 2008; 93 (6): 941-2. DOI: 10.3324/haematol.12460

14. Eldor A., Durst R., Hy-Am E., Goldfarb A., Gillis S., Rachmilewitz E.A., et al. A Chronic Hypercoagulable State in Patients with Beta-Thalassaemia Major Is Already Present in Childhood. Br J Haematol 1999; 107 (4): 739-46. DOI: 10.1046/j.13652141.1999.01758.x

15. Mullier F., Lainey E., Fenneteau O., Da Costa L., Schillinger F., Bailly N., et al. Additional Erythrocytic and Reticulocytic Parameters Helpful for Diagnosis of Hereditary Spherocytosis: Results of a Multicentre Study. Ann Hematol 2011; 90 (7): 759-68. DOI: 10.1007/ s00277-010-1138-3

16. Wagner G.M., Chiu D.T., Yee M.C., Lubin B.H. Red Cell Vesiculation - a Common Membrane Physiologic Event. J Lab Clin Med 1986; 108 (4): 315-24.

17. Troendle S.B., Adix L., Crary S.E., Buchanan G.R. Laboratory Markers of Thrombosis Risk in Children with Hereditary Spherocytosis. Pediatr Blood Cancer 2007; 49 (6): 781-5. DOI: 10.1002/pbc. 21319

18. Gelas T., Scalabre A., Hameury F., Dubois R., Grosos C., Mouriquand P.D., et al. Portal Vein Thrombosis after Laparoscopic Splenectomy during Childhood. J Thromb Thrombolysis 2014; 38 (2): 218-22. DOI: 10.1007/s11239013-1037-2

19. Das A., Bansal D., Ahluwalia J., Das R., Rohit M. K., Attri S.V., et al. Risk Factors for Thromboembolism and Pulmonary Artery Hypertension Following Splenectomy in Children with Hereditary Spherocytosis. Pediatr Blood Cancer 2014; 61 (1): 29-33. DOl: $10.1002 / p b c .24766$

20. Haybar H., Shahrabi S., Rezaeeyan H., Shirzad R., Saki N. Endothelial Cells: From Dysfunction Mechanism to Pharmacological Effect in Cardiovascular Disease. Cardiovasc Toxicol 2019; 19 (1): 13-22. DOI: 10.1007/s12012-0189493-8

21. Özdemir Z.C., Kar Y.D., Gündüz E., Bör Ö. Evaluation of the Coagulation Profile With Rotational Thromboelastometry in Children With Hereditary Spherocytosis. J Pediatr Hematol Oncol 2020; 42 (4): e195-8. DOI: 10.1097/ MPH.0000000000001702

22. Seregina E.A., Poletaev A.V., Bondar E.V., Vuimo T.A., Ataullakhanov F.I., Smetanina N.S. The Hemostasis System in Children with Hereditary
Spherocytosis. Thromb Res 2019; 176: 11-7. DOI: 10.1016/j.thromres.2019.02.004

23. Сметанина Н.C., Масчан А.А. Кузьминова Ж.А., Луговская С.А. Клинические рекомендации. Детская гематология. Под ред. Румянцева А.Г., Масчана А.А., Жуковской Е.В. М.: ГЭОТАР-Медиа; 2015. С. 145-59

24. Nasimuzzaman M., Arumugam P.I. Mullins E.S., James J.M., Vanden Heuvel K., Narciso M.G., et al. Elimination of the Fibrinogen Integrin AMB2-Binding Motif Improves Renal Pathology in Mice with Sickle Cell Anemia. Blood Adv 2019; 3 (9): 1519-32. DOl: 10.1182/bloodadvances.2019032342

25. Favaloro E.J., (Adcock) Funk D.M., Lippi G. Pre-Analytical Variables in Coagulation Testing Associated With Diagnostic Errors in Hemostasis. Lab Med 2012; 43 (2): 1.2-10. DOl: 10.1309/LM749BQETKYPYPVM

26. Постникова Л.Б. Кубышева Н.И. Миндубаев Р.3., Болдина М.В., Соодаева С.К. Особенности содержания эндотелина-1 и эндобронхиальной концентрации метаболитов оксида азота при хронической обструктивной болезни легких. Пульмонология 2010; 3: 108-12.

27. Frei A.C., Guo Y., Jones D.W., Pritchard K.A., Fagan K.A., Hogg N., et al. Vascular Dysfunction in a Murine Model of Severe Hemolysis. Blood 2008; 112 (2): 398-405. DOI: 10.1182/ blood-2007-12-126714

28. Iolascon A., Andolfo I., Barcellini W., Corcione F., Garçon L., De Franceschi L., et al. Recommendations Regarding Splenectomy in Hereditary Hemolytic Anemias. Haematologica 2017; 102 (8): 1304-13. DOI: 10.3324/ haematol.2016.161166

29. Alexakis N., Dardamanis D., Albanopoulos K., Ptohis N., Skalistira M., Karagiorga M., et al. Incidence Risk Factors and Outcome of Portal Vein Thrombosis After Laparoscopic-Assisted Splenectomy in $\beta$-Thalassemia Patients: A Prospective Exploratory Study. J Laparoendosc Adv Surg Tech 2013; 23 (2): 123-8. DOI: 10.1089/lap.2012.0268

30. Satitthummanid S., Uaprasert N., Songmuang S.B., Rojnuckarin P., Tosukhowong P., Sutcharitchan P., et al. Depleted Nitric Oxide and Prostaglandin E2 Levels Are Correlated with Endothelial Dysfunction in $\beta$-Thalassemia/HbE Patients. Int $\mathrm{J}$ Hematol 2017; 106 (3): 366-74. DOI: 10.1007/ s12185-017-2247-8

31. Gursel O., Tapan S., Sertoglu E., Taşçılar E., Eker I., Ileri T., et al. Elevated Plasma Asymmetric Dimethylarginine Levels in Children with Beta-Thalassemia Major May Be an Early Marker for Endothelial Dysfunction. Haematology 2018; 23 (5): 304-8. DOI: $10.1080 / 10245332.2017 .1396027$ 